\title{
ON $r$-REGULAR CONVERGENCE
}

\author{
PAUL A. WHITE
}

In his paper On sequences and limiting sets $[1],{ }^{1} \mathrm{G}$. T. Whyburn introduced the notion of regular convergence. He showed that in the cases of 0 and 1 regular convergence (see definition below) that the limit of sequences of many simple topological sets is of the same type as the members of the sequence. It is the purpose of this paper to extend some of these results to higher dimensions. The lack of simple characterizations of the higher dimension sets (such as the $n$-sphere) makes the results much weaker than in the 0 and 1 dimensional cases.

It is assumed throughout the paper that all sets lie in a compact metric space. All our complexes and cycles will be non-oriented, and the Vietoris cycles and chains ( $V$-cycles and $V$-chains) will have these as coordinates. The set of all points $x$ whose distance from a set $A$ is less than $\epsilon$ will be denoted by $U_{\epsilon}(A)$. Finally we shall denote the boundary of an $r$-dimensional complex (or $V$-chain) $z^{r}$ by $\dot{z}^{r}$.

Definition. $A$ sequence of closed sets $\left(A_{i}\right)$ converging to a limit set $A$ is said to converge $r$-regularly $(\rightarrow r)$ if for every $\epsilon>0$ there exist numbers $\delta>0$ and $N>0$ such that, if $n>N$, any $r$-dimensional $V$-cycle in $A_{n}$ of diameter less than $\delta$ is $\sim 0$ in a subset of $A_{n}$ of diameter less than $\epsilon$. If $A_{i} \rightarrow s A$ for all $s \leqq r$, we write $A_{i} \rightarrow \leqq r A[1]$.

Definition. $A$ Vietoris cycle $\xi^{r}=\left(x_{i}^{r}\right)$ is called a projection cycle if $\lim _{i \rightarrow \infty}$ (point set $\left.x_{i}^{r}\right)=X$ and each $x_{i}^{r} \subset X$. Clearly $X$ is the smallest carrier [2] of $\xi^{r}$.

Note. Corresponding to any cycle $\xi^{r}=\left(x_{i}^{r}\right)$ of a compact set $F$, there always exists a projection cycle $\xi_{1}^{r} \sim \xi^{r}$ in $F$. In fact if a convergent subsequence of $\left(x_{i}^{r}\right)$ is chosen, this set can be used as the set $X$ of the definition.

ThEOREM 1. If $A_{i} \rightarrow r A$, then for any $\epsilon>0$ there exist positive numbers $\delta$ and $N$ such that if $x^{r+1}$ is a simplex of $A_{i}(i>N)$ whose boundary has $a V$-chain realization [3] of diameter less than $\delta$, then $x^{r+1}$ has a V-chain realization of diameter less than $\epsilon$.

Proof. Let $\delta$ and $N$ be the numbers corresponding to $\epsilon$ in the definition of $r$-regular convergence and consider a simplex $x^{r+1}$ of $A_{i}(i>N)$

Presented to the Society, September 13, 1943; received by the editors September 30, 1943.

${ }_{1}$ Numbers in brackets refer to the bibliography at the end of the paper. 
whose boundary has a $V$-chain realization $\eta^{r}=\left(y_{i}^{r}\right)$ of diameter less than $\delta$. By the choice of $\delta$ and $N, \eta^{r} \sim 0$ in a subset of $A_{i}$ of diameter less than $\epsilon$. Thus there is a $V$-chain $\xi^{r+1}=\left(x_{i}^{r+1}\right)$ of diameter less than $\epsilon$ such that $\dot{x}_{i}^{r+1}=y_{i}^{r}$. Clearly $\xi^{r+1}$ is the $V$-chain realization of $x^{r+1}$ of diameter less than $\epsilon$.

THEOREM 2. If $A_{i} \rightarrow \leqq r . A$ then for any $\epsilon>0$ there exist positive numbers $\delta^{r}(\epsilon)$ and $N^{r}(\epsilon)$ such that if $x^{s}(s \leqq r+1)$ is a $\delta^{r}(\epsilon)$-simplex of $A_{i}$ $\left(i \geqq N^{r}(\epsilon)\right)$, then $x^{s}$ has a V-chain realization in a subset of $A_{i}$ of diameter less than $\epsilon$.

Proof. The proof shall be by induction.

The case $r=0$ is clearly a direct consequence of the definition of 0 -regular convergence where the numbers $\delta^{0}(\epsilon)$ and $N^{0}(\epsilon)$ are the numbers $\delta$ and $N$ of the definition.

Suppose the theorem to be true for $r=k-1$ and consider the case $r=k$. Let $\epsilon$ be an arbitrary positive number and $\delta<\epsilon, N$ be the numbers corresponding to it in. Theorem 1 . Now by hypothesis there exist positive numbers $\delta^{k-1}(\delta / 3)$ and $N^{k-1}(\delta / 3)$, and we define $\delta^{k}(\epsilon)=\min \left(\delta^{k-1}(\delta / 3), \quad \delta / 3\right)$ and $N^{k}(\epsilon)=N^{k-1}(\delta / 3)+N$. Let $x^{s}$ $(s \leqq k+1)$ be a $\delta^{k}(\epsilon)$-simplex of $A_{i}\left(i>N^{k}(\epsilon)\right)$. If $s \leqq k$, we know that $x^{s}$ has a $V$-chain realization of diameter less than $\delta / 3<\epsilon$ since $\delta^{k}(\epsilon) \leqq \delta^{k-1}(\delta / 3)$ and $N^{k}(\epsilon)>N^{k-1}(\delta / 3)$. If $s=k+1$, the boundary of $x^{k+1}$ is a $k$-dimensional $\delta^{k-1}(\delta / 3)$-cycle of $A_{i}\left(i>N^{k-1}(\delta / 3)\right)$. Thus each simplex of this cycle has a $V$-chain realization in a subset of $A_{i}$ of diameter less than $\delta / 3$. Furthermore we may suppose these realizations to be chosen so that common sides of two simplices have the same realizations. Now adding these $V$-chains for the simplices of the cycle we obtain a sequence of $k$-dimensional cycles, a subsequence of which yields a $k$-dimensional $V$-cycle of diameter less than $\delta^{k}(\epsilon)$ $+2(\delta / 3)<\delta / 3+2(\delta / 3)=\delta$ which is clearly a realization of the boundary of $x^{k+1}$. Now by the choice of $\delta$, we know that $x^{k+1}$ has a $V$-chain realization in a subset of $A_{i}\left(i>N^{k}(\epsilon)>N\right)$ of diameter less than $\epsilon$. Thus the theorem is true for $r=k$ and hence for all cases.

Theorem 3. If $A_{i} \rightarrow \leqq(r-1) A$ and $C$ is the smallest carrier of an essential projection cycle $\xi^{r}=\left(x_{j}^{r}\right)$ of $A$, then $C$ can be expressed as $\lim _{i \rightarrow \infty} C_{i}$ where $C_{i}$ is the smallest carrier of a cycle $\xi_{i}^{r}$ of $A_{i}$ which will be essential for all sufficiently large $i$.

Proof. Since $\xi r$ is essential, there is a positive number $\eta$ such that $\xi$ is not $\sim_{\eta} 0$ in $C$. Let $\epsilon_{k} \rightarrow 0$ be a sequence of positive numbers and let $3 \delta_{k}=\min \delta^{r-1}\left(\epsilon_{k} / 3\right), \delta^{r-1}(\eta / 3)$ (from Theorem 2). Choose $N_{k}^{\prime}$ such that for $i>N_{k}^{\prime}$ we have $U_{\delta_{k}}\left(A_{i}\right) \supset A, U_{\delta_{k}}(A) \supset A_{i}$ and let $N_{k}^{\prime \prime}=N_{k}^{\prime}$ 
$+N^{r-1}\left(\delta_{k} / 3\right)+N^{r-1}(\eta / 3)$. This defines a number $N_{k}^{\prime \prime}$ for each $k$, and by letting $N_{k}=\sum_{j=1}^{k} N_{j}^{\prime \prime}$ we obtain a monotone increasing sequence of numbers. Consider a $\delta_{k}$-cycle $x_{j(k)}^{r}$ of $\xi^{r}$ that is not $\sim_{\eta} 0$ in $C$ and such that $U_{\delta_{k}}\left(x_{j(k)}^{r}\right) \supset C$. Now pick a number $n>N_{k}$ and let $a_{0}, a_{1}, \cdots, a_{g}$ be the vertices of $x_{j(k)}^{r}$, then for each $s \leqq g$, let $b_{s}$ be a point of $A_{n}$ such that $\rho\left(a_{s}, b_{s}\right)<\delta_{k}$. For each simplex $\left(a_{i_{0}}, a_{i_{1}}, \cdots, a_{i_{r}}\right)$ in $x_{j(k)}^{r}$ let $\left(b_{i_{0}}, b_{i_{1}}, \cdots, b_{i_{r}}\right)$ be a simplex and let $x^{r}$ be the cycle consisting of these simplices. Clearly $x^{r}$ will be a $3 \delta_{k}$-cycle of $A_{n}$, and we shall call any cycle obtained in this manner a $\delta_{k}$-projection of $x_{j(k)}^{r}$ [1]. Our choice of $3 \delta_{k}$ allows us to realize each simplex of $x^{r}$ in a subset of $A_{n}$ of diameter less than $\epsilon_{k} / 3, \eta / 3$, from which we obtain a $V$-cycle realization $\xi_{n}^{r}=\left(x_{j n}^{r}\right)$ of $x^{r}$ in $U_{\delta_{k} / 3}\left(x^{r}\right)$ as well as in $U_{\eta / 3}\left(x^{r}\right)$. Now $C_{n}$, the smallest carrier of $\xi_{n}^{r}$, will satisfy the conditions of our theorem, for $C_{n} \subset \bar{U}_{\epsilon_{k} / 3}\left(x^{r}\right), x^{r} \subset U_{\delta_{k}}\left(x_{j(k)}^{r}\right), x_{j(k)}^{r} \subset U_{\delta_{k}}\left(x^{r}\right), C \subset U_{\delta_{k}}\left(x_{j(k)}^{r}\right)$. Therefore, $C_{n} \subset U_{\epsilon_{k}}(C), C \subset U_{\epsilon_{k}}\left(C_{n}\right)$ as $\left(\epsilon_{k} / 3\right)+\delta_{k}+\delta_{k}<\epsilon_{k} / 3+\epsilon_{k} / 3+\epsilon_{k} / 3=\epsilon_{k}$. Now for all $n$ such that $N_{k} \leqq n<N_{k+1}$, choose $C_{n}$ corresponding to $\epsilon_{k}$; then $\lim _{n \rightarrow \infty} C_{n}=C$.

Finally, since $\xi_{n}^{r} \subset U_{\eta / 3}\left(x^{r}\right)$, we obtain by an application of the prism construction [4] the homology $x_{j n}^{r} \sim_{\eta / 3} x^{r}$ for all $j$. Thus $\xi_{n}^{r}$ is not $\sim_{\eta / 3} 0$ in $C_{n}$, for if it were then $x^{r} \sim_{\eta / 3} 0$; but by a $\delta_{k}$-projection into $C$ of the $\eta / 3$-complex bounded by $x^{r}$ we could obtain an $\eta$-complex in $C$ bounded by $x_{j(k)}^{r}$, contrary to our hypothesis. This shows that $\xi_{n}^{r}$ is essential for sufficiently large $n$ and concludes the proof.

The necessity of the regular convergence in the preceding theorem is shown by the following example. Let $A_{i}$ be the arc of the circle $\rho=1$ where $\theta$ varies from $1 / i$ to $2 \pi-(1 / i)$; then $A$ will be the circle $\rho=1$, and the 0 -regular convergence is clearly violated. Now $A$ is the smallest carrier of an essential 1-cycle, but the theorem cannot be satisfied, as $A_{i}$ contains no essential 1-cycles.

CoRollary 3.1. If $A_{i} \rightarrow \leqq(r-1) A$, where $A_{i}$ is a $T_{r}$-set [5] for each $i$, then $A$ is a $T_{r}$-set.

PROoF. If the theorem were not true, then $T$ would contain an essential $r$-dimensional cycle; but by the theorem there would exist essential $r$-dimensional cycles in some of the $A_{i}$, which contradicts their property of being $T_{r}$-sets.

THEOREM 4. If $M_{i} \rightarrow \leqq r M$ and $B$ is an irreducible membrane [2] for the homology $\xi^{r}=\left(x_{j}^{r}\right) \sim 0$ in $M$, then $B$ can be expressed as $\lim _{i \rightarrow \infty} B_{i}=B$, where $B_{i} \subset M_{i}$ is an irreducible membrane for a homology $\xi_{i}^{r} \sim 0$ in $M_{i}$.

Proof. Let $\epsilon_{k} \rightarrow 0$ be a sequence of positive numbers and let $3 \delta_{k}=\delta^{r}\left(\epsilon_{k}\right)$ and $N^{r}\left(\epsilon_{k}\right)$ be the numbers corresponding to $\epsilon_{k}$ in Theorem 
2. Choose $N_{k}^{\prime}$ such that for $i>N_{k}^{\prime}, U_{\delta_{k}}\left(M_{i}\right) \supset M, U_{\delta_{k}}(M) \supset M_{i}$, and let $N_{k}^{\prime \prime}=N^{r}\left(\epsilon_{k}\right)+N_{k}^{\prime}$. This defines a number $N_{k}^{\prime \prime}$ for each $k$, and by letting $N_{k}=\sum_{j=1}^{k} N_{j}^{\prime \prime}$ we obtain a monotone increasing sequence of numbers. Since $B$ is an irreducible membrane of $\xi^{r} \sim 0$ in $M$, we can find a $V$-chain $\eta^{r+1}=\left(y_{j}^{r+1}\right)$ in $M$ such that $j_{j}^{r+1}=x_{j}^{r}$ for each $j$, and such that the point sets $y_{j}^{r+1}$ converge to $B$. Let $j(k)$ be chosen such that $y_{j(k)}^{r+1}$ is a $\delta_{k}$-complex and $U_{\epsilon_{k}}\left(y_{j(k)}^{r+1}\right) \supset B, U_{\epsilon_{k}}(B) \supset y_{j(k)}^{r+1}$. Let $n$ be any fixed number greater than $N_{k}$, and project $y_{j(k)}^{r+1}$ by means of a $\delta_{k}$-projection into a $\left(3 \delta_{k}\right)$-complex $y^{r+1}$ of $M_{n}$. Since $3 \delta_{k}=\delta^{r}\left(\epsilon_{k}\right)$, each simplex of $y^{r+1}$ has a $V$-chain realization in a subset of $M_{n}$ of diameter less than $\epsilon_{k}$. Combining these realizations for all simplices of $y^{r+1}$, we obtain a $V$-chain realization $\eta_{n}^{r+1}=\left(y_{n}^{r+1}\right)$ of $y^{r+1}$ in $U_{\epsilon_{k}}\left(y^{r+1}\right)$. Now a $V$-cycle can be formed from a subsequence of $\left(\dot{y}_{j n}^{r+1}\right)$, and a further subsequence can be chosen so that the remaining $\left(y_{j n}^{r+1}\right)$ converge to a set $B_{n}{ }^{\prime}$. We shall denote this subsequence by the same notation $\eta_{n}^{r+1}=\left(y_{j n}^{r+1}\right)$ and we shall let $\xi_{n}^{r}=\left(\dot{y}_{j n}^{r+1}\right)$. Let $B_{n} \subset B_{n}^{\prime}$ be an irreducible membrane of the homology $\xi_{n}^{r} \sim 0$ in $M_{n}$. Now $U_{\epsilon}(B) \supset y_{j(k)}^{r+1}, \quad U_{\delta_{k}}\left(y_{j(k)}^{r+1}\right)^{r} \supset y^{r+1}, \quad \bar{U}_{\epsilon_{k}}\left(y^{r+1}\right) \supset B_{n}^{\prime} \supset B_{n}, \quad B_{n} \supset y^{r+1}$ $U_{\delta_{k}}\left(y^{r+1}\right) \supset y_{j(k)}^{r+1}, U_{\delta_{k}}\left(y_{j(k)}^{r+1}\right) \supset B$; therefore $U_{3 \epsilon_{k}}(B) \supset B_{n}$ and $U_{3 \epsilon_{k}}\left(B_{n}\right)$ $\supset B$. Thus if we choose $\xi_{n}^{r}$ and $B_{n}$ in $M_{n}$ corresponding to $\epsilon_{k}$ for all $n$ such that $N_{k} \leqq n<N_{k+1}$, we shall have $\lim _{n \rightarrow \infty} B_{n}=B$, and the conclusion of the theorem.

The necessity of the regular convergence in the preceding theorem is shown by allowing $M_{i}$ to be a totally disconnected set for each $i$ such that $\lim _{i \rightarrow \infty} M_{i}=M$ is a unit interval, and hence the irreducible membrane of the homology of the 0-cycle consisting of its end points. Now clearly the convergence is not 0 -regular, and the conclusion of the theorem is violated since no 0 -cycle of any $M_{i}$ is $\sim 0$.

Corollary 4.1. If $M_{i} \rightarrow \leqq r M$ and $A_{i} \rightarrow A$ where $A_{i}$ is an $A_{r}$-set [5] of $M_{i}$ for each $i$, then $A$ is an $A_{r}$-set of $M$.

Proof. Consider any irreducible membrane $B$ of the homology $\xi^{r} \sim 0$ in $M$ where $\xi^{r} \subset A$. By Theorem 4 there exist cycles $\xi_{i}^{r}$ in $M_{i}$ for each $i$ and irreducible membranes $\left(B_{i}\right)$ of the homologies $\xi_{i}^{r} \sim 0$ in $M_{i}$, such that $B_{i} \rightarrow B$. Also since $A_{i} \rightarrow A$ and $\xi^{r} \subset A$, we can choose a $\xi_{i}^{r}$ in $A_{i}$ for each $i$. By the definition of an $A_{r}$-set we have $B_{i} \subset A_{i}$ for each $i$; therefore $B=\lim _{i \rightarrow \infty} B_{i} \subset \lim _{i \rightarrow \infty} A_{i}=A$. Thus $A$ is an $A_{r}$-set of $M$.

Theorem 5. If $C_{i} \rightarrow \leqq r C$, where $C_{i}$ is the irreducible carrier of an $r$-dimensional projection cycle $\xi_{i}^{r}$ for each $i$, then $C$ is the irreducible carrier of a projection cycle $\xi^{r}$. Finally $\xi^{r}$ will be essential if and only if all but a finite number of the $\xi_{i}^{r}$ are essential. 
Proof. Corresponding to $\delta_{i} \rightarrow 0$, we can pick a subsequence of $\left(C_{i}\right)$, which we shall suppose to be the whole sequence, such that $U_{\delta_{i} / 3}\left(C_{i}\right) \supset C, U_{\delta_{i} / 3}(C) \supset C_{i}$. Let $\xi_{i}^{r}=\left(x_{i j}^{r}\right)$; then there exists a $\delta_{i} / 3$ cycle $x_{i n_{i}}^{r}$ such that $U_{\delta_{i} / 3}\left(x_{i_{i}}^{r}\right) \supset C_{i}, U_{\delta_{i} / 3}\left(C_{i}\right) \supset x_{i n_{i}}^{r}$. Project $x_{i n_{i}}^{r}$ into a $\delta_{i}$-cycle $x_{i}^{r}$ of $C$. Clearly $U_{\delta_{i}}\left(x_{i}^{r}\right) \supset C, U_{\delta_{i}}(C) \supset x_{i}^{r}$. Pick a subsequence of $\left(x_{i}^{r}\right)$, which we shall suppose to be the whole sequence, forming a $V$-cycle $\xi^{r}$. Now the point sets $x_{i}^{r} \rightarrow C$ and $\xi^{r}=\left(x_{i}^{r}\right) \subset C$; therefore $C$ is the irreducible carrier of $\xi^{r}$.

Now suppose that $\xi^{r}$ is not essential, then $\xi^{r} \sim 0$ in $C$. Since $C_{i} \rightarrow \leqq r C$, we know by a theorem of G. T. Whyburn [7] that $C$ is an $l c^{r}$; hence by a theorem of R. L. Wilder [3] the $r$-dimensional Betti number of $C$ is a finite number $n$. A result of $H$. A. Arnold [8] implies that all but a finite number of the $C_{i}$ have this same finite Betti number. Let $\xi_{10}^{r}, \cdots, \xi_{n 0}^{r}$ be a basis for $r$-dimensional cycles in $C$, which we can choose to be projection cycles with smallest carriers $C_{01}, \cdots, C_{0 n}$. By Theorem 3 there exist cycles $\xi_{11}^{r}, \cdots, \xi_{n t}^{r}$ with smallest carriers $C_{1 i}, \cdots, C_{n i}$ in $C_{i}$ such that $\lim _{i \rightarrow \infty} C_{j i}=C_{j}$ $(j=1, \cdots, n)$. The $\left(\xi_{j t}^{r}\right)$ will be linearly independent for $i$ greater than some integer $N_{1}$, for if not we can establish by a projection a linear dependence of the $\left(\xi_{j 0}^{r}\right)$. Since the Betti number of each $C_{i}$ for $i$ greater than some number $N_{2}$ is $n$, it follows that $\left(\xi_{j l}^{r}\right)$ is a basis for cycles in $C_{i}$ for $i>N=N_{1}+N_{2}$. Thus $\xi_{i}^{r} \sim \sum_{j=1}^{n} \alpha_{j} \xi_{j \ell}^{r}(i>N)\left(\alpha_{j}=0\right.$ or 1$)$, where we can suppose that $N$ was chosen large enough so that the same linear combination holds for each $i$. Now by projecting the complexes bounded by $\xi_{i}^{r}+\sum_{j=1}^{n} \alpha_{j} \xi_{j t}^{r}$, we can establish a homology $\xi^{r} \sim \sum_{j=1}^{n} \alpha_{j} \xi_{j}^{r}$. But $\xi^{r} \sim 0$; therefore $\alpha_{j}=0$ for all $j$. Thus $\xi_{i}^{r} \sim 0$ in $C_{i}$ for $i>N$, which implies that $\xi_{i}^{r}$ is inessential.

Conversely, if $\xi^{r}$ is essential then exactly the same procedure as was used in Theorem 3 can be used to show that all but a finite number of the $\xi_{\imath}^{r}$ are essential.

The necessity of the regular convergence in the preceding theorem is shown by the following example. Let $C_{i}$ be the collection of points $\left(x=j / 3^{i}, y=0\right)$ for $j=0,1, \cdots, 3^{i}$, then $\lim _{i \rightarrow \infty} C_{i}=C=$ the unit interval from 0 to 1 , and clearly the 0 -regular convergence is violated. Now each $C_{i}$ is an essential 0-dimensional $V$-cycle and hence is its own irreducible carrier, but $C$ clearly cannot be the irreducible carrier of an essential 0 -cycle as all 0 -cycles are $\sim 0$ in $C$.

THEOREM 6. If $B_{i} \rightarrow \leqq r B$, where $B_{i}$ is an irreducible membrane for an homology of a projection cycle $\xi_{i}^{r} \sim 0$ in $B_{i}$ for each $i$, then $B$ is an irreducible membrane of an homology of a projection cycle $\xi^{r} \sim 0$ in $B$.

Proof. In the proof of Theorem 5 we have seen how to establish a projection cycle $\xi^{r}$ in $B$. Furthermore by projections into $B$ of the 
chains bounded by the $\xi_{i}$, we can establish an homology $\xi^{r} \sim 0$ in $B$. It remains to show that $\xi^{r}$ is not $\sim 0$ in a proper subset $B^{\prime}$ of $B$. To this end suppose $\xi^{r} \sim 0$ in $B^{\prime}$. By Theorem $4, B^{\prime}=\lim _{i \rightarrow \infty} B_{i}^{\prime}$ where $B_{i}^{\prime}$ is an irreducible membrane of the homology $\xi_{i}^{r} \sim 0$ in $B_{i}$. (Since the carrier of $\xi^{r}$ was chosen as the limit of the carriers of the $\xi_{i}^{r}$, we can choose the $B_{i}^{\prime}$ corresponding to the homologies of our original $\xi_{i}^{r}$.) Thus $B^{\prime}=\lim _{i \rightarrow \infty} B_{i}^{\prime}=\lim _{i \rightarrow \infty} B_{i}=B$ and $B$ is an irreducible membrane of $\xi^{r} \sim 0$ in $B$.

THEOREM 7. If $M_{i}^{r} \rightarrow \leqq r M$, where $M_{i}^{r}$ is an r-dimensional closed Cantorian manifold [6] for each $i$, then if $\operatorname{dim} M \leqq r, M$ is also a closed $r$-dimensional Cantorian manifold.

Proof. Since $p^{r}\left(M_{i}^{r}\right) \neq 0$ for each $i\left(p^{r}\left(M_{i}^{r}\right)=\right.$ the $r$ th dimensional Betti number of $M_{i}^{r}$ ) and the convergence is regular, it follows that $p^{r}(M) \neq 0$. Next suppose $M^{\prime}$ is a proper closed subset of $M$ with $p^{r}\left(M^{\prime}\right) \neq 0$. Then there exists an essential (projection) cycle $\xi^{r}$ with irreducible carrier $C$ in $M^{\prime}$. By Theorem $3, C=\lim _{k \rightarrow \infty} C_{k}$, where $C_{k}$ is the smallest carrier of an essential cycle $\xi_{k}^{r}$ in $M_{k}^{r}$. Now for some integer $n, C_{n}$ will be a proper closed subset of $M_{n}^{r}$, but by the definition of $M_{n}^{r}, p^{r}\left(C_{n}\right)=0$ contrary to the fact that $\xi_{n}^{r}$ is essential. Thus $p^{r}\left(M^{\prime}\right)=0$ for every proper closed subset $M^{\prime}$ of $M$. Finally $\operatorname{dim} M \geqq r$, for $p^{r}(M) \neq 0$, implies the existence of an essential $\xi^{r}$ in $M$. Since by hypothesis $\operatorname{dim} M \leqq r$, we have $\operatorname{dim} M=r$, and $M$ is an $r$-dimensional closed Cantorian manifold.

\section{BIBLIOGRAPHY}

1. G. T. Whyburn, On sequences and limiting sets, Fund. Math. vol. 25 (1935) pp. 408-426.

2. R. L. Wilder, Unified analysis situs, Bull. Amer. Math. Soc. vol. 38 (1932) pp. 649-692.

3. - On locally connected spaces, Duke Math J. vol. 1 (1935) pp. 543-555.

4. P. Alexandroff and H. Hopf, Topologie, Berlin, 1935, p. 199.

5. G. T. Whyburn, Cyclic elements of higher order, Amer. J. Math. vol. 56 (1934) pp. 133-146.

6. P. Alexandroff, Gestalt und Lage, Ann. of Math. vol. 30 (1928) pp. 101-187.

7. G. T. Whyburn, Regular convergence and monotone transformations, Amer. J. Math. vol. 57 (1935) pp. 902-906.

8. H. A. Arnold, On r-regular convergence of sets, Bull. Amer. Math. Soc. Abstract 47-7-326.

Louisiana State University 